\title{
Emotions, reasoning, and mental health as predictors of behavior during three phases of the COVID-19 pandemic
}

\author{
Yanick Leblanc-Sirois $^{1}$, Marie-Ève Gagnon ${ }^{2}$ and Isabelle Blanchette ${ }^{1}$ \\ ${ }^{1}$ Université Laval ${ }^{2}$ Université du Québec à Trois-Rivières \\ Contact author: Yanick.leblanc-sirois.1@ulaval.ca
}

Working paper, December 10, 2020

\begin{abstract}
The COVID-19 pandemic has required people worldwide to adjust their behavior for several months in response to a crisis of rare proportions. Little is known about the specific factors that affected the progression of the public's reactions during the pandemic. Individual factors associated with pandemic-related behavior in general, and compliance with public health measures in particular, are not firmly established. We undertook a survey of behavior, emotions, reasoning style, and mental health in the province of Quebec at the beginning, the peak, and the aftermath of the first wave of the COVID-19 pandemic. We recruited 530 responders from a convenience sample; 154 responders participated in all three surveys. Emotions were most intense at the beginning of the first wave of the pandemic, not at its peak. Responders' compliance with three public health measures decreased between the peak and the aftermath of the first wave of the pandemic; however, mask wearing also became more common. Pandemicrelated behavior in general, and compliance with public health measures specifically, were predicted by avoidance-related emotions evoked by the pandemic. Approach-related emotions linked to the societal response contributed specifically to the prediction of compliance with public health measures. In contrast, reasoning style and mental health did not as consistently predict behavior during the pandemic. Our research may help inform public health policy during other waves of the COVID-19 pandemic and future global health crises.
\end{abstract}

Keywords: coronavirus, emotions, reasoning, mental health 


\section{Introduction}

On March $11^{\text {th }}, 2020$, the World Health Organisation announced that the spread of the novel coronavirus SARS-CoV-2 had given rise to a global pandemic. Governments across the globe implemented emergency public health measures in order to slow down the spread of COVID-19. In the province of Quebec, public schools and universities were shut down on March $13^{\text {th }}$. Additional emergency measures were announced in the following days and weeks. Public health campaigns urged people to modify their behavior and comply with public health measures by washing hands regularly, avoiding touching one's face, and practicing social distancing.

Varying degrees of compliance with public health measures, from active disrespect to complete compliance, were observed in the population. Moreover, some people changed their behavior in ways that were not recommended, such as buying toilet paper in bulk or increasing their intake of dietary supplements. A better understanding of the individual factors that predisposed people to adopt different behaviors during the first wave of the pandemic could help organisations adjust public health campaigns during future global health crises. Individual differences in the intensity of emotions experienced, reasoning style, and mental health symptomatology are possible predictors of behavior during crises such as the COVID-19 pandemic. Moreover, a descriptive account of the progression of behavior, emotion and mental health during diverse phases of the pandemic may help predict the progression of behavior during current and future global health crises.

\section{Emotions and behavior during the COVID-19 pandemic}


Individual differences in emotional reactions may provide part of the explanation for individual differences in behavior during the COVID-19 pandemic. The approach/avoidance motivation model provides a theoretical framework which proposes causal effects of emotions on behavior. According to this model, emotions can influence the decision-making process by providing motivation towards approaching or avoiding real or anticipated stimuli (Elliot et al., 2013). Positive emotions are generally linked to approach motivation and most negative emotions are generally linked to avoidance motivation. This model suggests that negative emotions such as fear and anxiety could increase avoidance of COVID-19 and may thus lead to more pandemicrelated behavior in general, including more compliance with public health measures. At the same time, positive emotions evoked by the societal response could increase active participation in this response and specifically encourage compliance with public health measures. Supporting this prediction, higher levels of fear evoked by COVID-19 were a strong predictor of more behavior change during the COVID-19 pandemic (Harper et al., 2020). Moreover, a higher level of positive emotions evoked by the societal response at the beginning of the pandemic predicted higher levels of compliance with public health measures (Bogg \& Milad, 2020). However, these previous studies only investigated the links between behavior and emotions during one phase of the pandemic. Therefore, the temporal consistency of the link between emotions and behavior throughout the pandemic has yet to be studied.

\section{Progression of emotions during the pandemic}

If emotions influence behavior during the pandemic, then the continued presence of behavior related to COVID-19 may depend in part on the progression of the intensity of emotions. Frijda (1988) has proposed a widely cited set of general rules ("laws") that can help predict the progression of emotions elicited by an event. These general rules can be applied to predict how 
emotions progress as the COVID-19 pandemic unfolds. Frijda's rule of "apparent reality" states that the intensity of emotions should be proportional to the degree to which the events elicited by the emotions appear or are appraised as real. Following this general rule, we might expect emotions to be at their strongest at the peak of the first wave of the pandemic, when the number of active cases and deaths is highest. However, Frijda's rule of habituation states that emotions tend to become less intense with repeated exposure to the event that elicits emotions. According to this rule, we might expect the peak of emotional intensity to occur shortly after the declaration of a global pandemic and the onset of a province-wide lockdown, when most people are exposed to the idea of a global pandemic for the first time. Because the pandemic's first wave had its peak several weeks after its onset, there is an apparent conflict between these two predictions. Thus, an empirical investigation is necessary to characterize the progression of emotions during the pandemic.

Previous empirical studies of the progression of emotions have shown that the duration of an emotion correlates positively with the initial intensity of the eliciting events (Verduyn, 2009), that a reappearance of the event or rumination about the event could extend this duration (Verduyn, 2009; Verduyn \& Lavrijsen, 2015), that longer-lasting events elicit longer-lasting emotions (Verduyn et al., 2015), and that events of high importance elicit longer-lasting emotions (Verduyn et al., 2015; Verduyn \& Lavrijsen, 2015b). Together, these studies suggest that emotions linked to the COVID-19 pandemic can be expected to be long-lasting, but do not provide insight about the phase of the pandemic when emotions can be expected to peak. Moreover, these studies relied on retrospective reports of remembered emotions that may not reflect how emotions were experienced at the time of the event (Levine \& Bluck, 1997). The 
progression of emotions may have implications for the progression of behavior during the pandemic.

\section{Reasoning and behavior during the COVID-19 pandemic}

Reasoning style can also influence behavior. Specifically, some decisions can be predicted from individual differences in people's tendency to reason intuitively or systematically (Calvillo \& Burgeno, 2015; Pennycook \& Rand, 2019). The literature on intuitive versus systematic reasoning is largely based on dual-process models of reasoning (Evans \& Stanovich, 2013), which propose a fundamental distinction between two types of reasoning. Type 1 processes require little use of cognitive resources and tend to be intuitive, effortless and fast. The use of heuristics in reasoning, for instance, is considered a Type 1 process. Type 1 processes are subject to cognitive biases that can lead to mistakes according to normative accounts of reasoning. In contrast, Type 2 processes require intensive use of cognitive resources such as working memory and tend to be systematic, effortful, and slow. Reasoning systematically from premises in order to reach a conclusion according to the rules of logic is an example of Type 2 reasoning. Everyone can make use of both types of processes, but there are reliable inter-individual differences in people's tendency to use one type of reasoning over the other (Primi et al., 2016; Stagnaro \& al., 2018).

There is a current debate about the relative usefulness of Type 1 versus Type 2 reasoning. The bounded rationality perspective suggests that heuristics are often adapted to their environment. According to this perspective, Type 1 processes often promote adaptive behavior at little cost in terms of cognitive resources, allowing these cognitive resources to be directed elsewhere (Todd \& Gigerenzer, 2012). An opposing view suggests that while Type 1 processes are generally useful, they are more likely to yield errors, especially in novel situations that result from 
important changes in the environment. According to this point of view, the previously useful heuristics may not be adapted to the new environment after such a change (Evans, 2010). The COVID-19 pandemic may be a prime example of such a novel situation. According to this perspective, overriding the default Type 1 reasoning in favor of Type 2 reasoning is likely to yield fewer mistakes in reasoning, and therefore result in behavior that is more adapted to the pandemic. This perspective suggests that Type 2 reasoning could encourage the adoption of evidence-based measures such as social distancing, more frequent hand washing and efforts not to touch one's face.

At the time of writing, there is mixed evidence about the impact of different types of reasoning on decision-making in the context of the COVID-19 pandemic. Some research suggests that Type 2 reasoning is linked to compliance with public health measures. Stanley and colleagues (2020) used the Cognitive Reflection Test (CRT) as a measure of responders' tendency to reason systematically (Type 2) rather than intuitively (Type 1). They reported a positive correlation between performance on the CRT and behaviors such as handwashing and social distancing. Greater working memory capacity, an important predictor of the ability to reason systematically (Stupple et al., 2013), also predicted higher compliance with public health measures (Xie et al., 2020). Moreover, instructing people to rely on reasoning rather than emotions increased participants' intentions of wearing a mask during the COVID-19 pandemic (Capraro \& Barcelo, 2020). However, mixed and null results have also been obtained. Scientific reasoning (a Type 2 process) was linked to the adoption of fewer ineffective COVID-19 mitigation measures such as drinking strong alcohol as a disinfectant, but also fewer effective COVID-19 mitigation measures such as hand washing and social distancing (Čavojová et al., 2020). Two studies found that indexes of Type 2 reasoning performance did not predict compliance with public health measures 
(Erceg et al., 2020; Pennycook et al., 2020). In summary, further studies are necessary to determine whether Type 2 reasoning increases compliance with public health measures during a pandemic more than Type 1 reasoning.

\section{Mental health and behavior during the COVID-19 pandemic}

The COVID-19 pandemic, along with associated public health measures such as social distancing, has an important impact on mental health. Empirical studies from several countries including China (Wang et al., 2020), Italy (Rossi et al., 2020) and the United States (Fitzpatrick et al., 2020; Twenge \& Joiner, 2020) indicate elevated rates of depression and generalized anxiety in the general population during the pandemic. Little is known about how mental health evolves over time during a pandemic, but one cross-sectional longitudinal study (Wang et al., 2020) offered evidence that mental health consequences of the pandemic became slightly less severe as time progressed. We sought to replicate this result.

Moreover, little is known about the links between behavior and mental health during the pandemic. It is possible that better mental health results in decision making that is more adapted to the situation. If this is the case, mental health symptomatology should be negatively associated with compliance with public health measures. On the other hand, it is possible that generalized anxiety and depression are in part caused by high anxiety evoked by the pandemic, which we have identified as a possible predictor of compliance with public health measures. In this case, generalized anxiety and depression symptoms may be associated with more compliance with public health measures.

Evidence linking behavior and mental health in the context of the COVID-19 pandemic is mixed. One study shows that an increase in depressive symptoms after the beginning of the pandemic 
was associated with lower compliance with public health measures (Solomou \& Constantinidou, 2020). However, two studies revealed that having more generalized anxiety and depression symptoms was associated with higher rates of compliance with public health measures such as hand washing, mask wearing and social distancing (Marroquín, 2020; Stickley et al., 2020). In view of these two reports, it is possible that interventions aiming to improve mental health might unintentionally reduce compliance with public health measures, though we note that no such causal effect has been demonstrated. Further exploration of mental health as a possible predictor of behavior during the pandemic might help predict the possible impacts of mental health interventions on behavior.

\section{$\underline{\text { Objectives }}$}

The first objective of the study was to document the progression of emotions, behavior and mental health across different phases of the first wave of the COVID-19 pandemic. We aimed to determine whether the progression of the intensity of emotions would follow a downwards trend as the pandemic unfolded, or whether the peak of the intensity of emotions would correspond to the peak number of active cases. We also aimed to study whether depression and generalized anxiety symptoms would become less common between the peak of the first wave of the pandemic and its aftermath.

The second objective of the study was to document the predictors of behavior change during the pandemic and to explore the roles of emotions, reasoning and mental health. We hypothesized that higher levels of avoidance-related emotions such as fear and anxiety would be associated with more pandemic-related behavior. We also hypothesized that approach-related positive emotions evoked by the societal response, (i.e. the way governments, public health organisations and individuals reacted towards the pandemic), would correlate specifically with compliance with 
public health measures. We also aimed to determine whether reasoning intuitively or systematically impacted behavior during the pandemic, and whether anxiety and depression symptoms were associated with compliance with public health measures.

\section{Method}

\section{$\underline{\text { Participants }}$}

One week after emergency measures were declared in the province of Quebec in response to the COVID-19 pandemic (March $13^{\text {th }}$ ?), we recruited a convenience sample on social media platforms for an online survey about behavior, emotions, reasoning, and mental health in the context of the COVID-19 pandemic. Participants were over 18 years of age, fluent in French, and living within the borders of the province of Quebec when they responded. Between March $20^{\text {th }}$ and $27^{\text {th }} 2020,530$ responders $(19.2 \%$ men, $79.2 \%$ women, $1.6 \%$ other $/$ no response; mean age $=$ $35.3, \mathrm{SD}=13.4)$ completed the first questionnaire. Of these, 224 responders $(28.7 \%$ men, $71 \%$ women) filled the second questionnaire between April $24^{\text {th }}$ and May $1^{\text {st }}$, when the number of new cases per day in Quebec was close to its maximum. At that time, the provincial government was also beginning to loosen some lock-down measures. A third questionnaire, accessible between June $26^{\text {th }}$ and July $3^{\text {rd }}$, received 171 responses from the responders who had filled the first questionnaire ( $25.1 \%$ men, $74.9 \%$ women). In total, 154 responders filled out all three questionnaires (29\% of total). Participants did not receive financial compensation. The research project was approved by the ethics committee at Université du Québec à Trois-Rivières.

Excess death statistics in the province of Quebec provide additional context about the timing of the three phases of our study. During the weeks corresponding to phase 1 (beginning of the pandemic) and phase 3 (aftermath of the first wave) of our study, fewer than 50 excess deaths 
were recorded in the province of Quebec in 2020 compared to an average of the same weeks in the previous two years. In contrast, over 750 excess deaths were recorded during the week corresponding to phase 2 (peak of the first wave) of our study (Institut de la statistique du Québec, 2020).

\section{Measures of primary interest}

Table 1 provides an overview of the three different questionnaires and all of their components. In this report, we provide a detailed description of all components that are relevant to the stated research questions.

Phase

Self-reported behavior ${ }^{1}$

Self-reported emotions

Cognitive reflection test

Deductive reasoning

Base rates

PHQ-9 and GAD-7

Sociodemographic ${ }^{3}$

Predicted impacts

checklist $^{3}$

Beliefs $^{3}$

Life situations ${ }^{3}$

Affective image

evaluation $^{3}$

Sources of information
1 - Beginning of the pandemic

$\mathrm{X}$

X

X

$\mathrm{X}^{2}$

$\mathrm{X}^{2}$

X

X

X

X

X
X

2-Peak of the

first wave

X

X

X

X

X
3 - Aftermath of the

first wave

X 
Opinion about return to

normal $^{3} \quad \mathrm{X}$

Table X. Overview of questionnaires in all three phases of the pandemic. ${ }^{1}$ Presence or absence of behavior in the first phase, frequency of behavior for phases 2 and 3. ${ }^{2}$ Sample was split between the deductive reasoning task and the base rate task. ${ }^{3}$ Measures not directly related to the main research questions are not detailed in the current report, but are reported here for full disclosure.

\section{Self-reported behavior}

We provided participants with a list of 12 behaviors that could be adopted in response to the COVID-19 pandemic and asked them to indicate which behaviors they adopted. Twelve behaviors were identified: washing hands often, avoiding touching one's face, practicing social distancing, using antibacterial gel, buying food in bulk, buying toilet paper in bulk, buying other health or cleaning products in bulk, consuming larger quantities of warm drinks, efforts to clean one's residence, voluntary isolation (not leaving home), wearing gloves or a mask, and increasing consumption of dietary supplements.

In the first questionnaire, we asked about the presence or absence of behaviors, rather than their frequency, because the time since the beginning of lockdown could be as short as one week. We deemed this duration insufficient to estimate the frequency of adopting each behavior. In phases two and three, the questionnaire was adjusted to measure the frequency of the same behaviors since the beginning of the pandemic (phase 2) and during the previous two weeks (phase 3). Consequently, the self-reported behavior measure in phase 1 cannot be directly compared with those in phases 2 and 3.

We computed a "pandemic-related behavior" score by summing the twelve items on the COVID19 behavior checklist. Some of these behaviors were effective and recommended by provincewide public health campaigns (e.g. social distancing), some were effective but not specifically 
recommended (e.g. use of antibacterial gel), while others were not recommended and ineffective (e.g. buying in bulk). Thus, the pandemic-related behavior score indexes the presence and frequency of all the pandemic-related behaviors that we measured, irrespective of their expected adaptive value.

The behaviors that were emphasized by public health campaigns in the province of Quebec throughout the first four months of the pandemic were to wash one's hands regularly with soap, avoid touching one's face, and practice social distancing (avoiding crowds and close contacts). We created a score of "compliance with public health measures" by adding presence/absence scores (phase 1) or frequency scores (phases 2 and 3) for these three behaviors. A higher score represented more compliance with public health measures. We note that mask-wearing was not included in this category: although mask-wearing had become strongly encouraged between phases 2 and 3 of our study, public health authorities did not encourage mask-wearing in the general population for several weeks after the beginning of the first wave of the pandemic.

\section{Self-reported emotions}

We asked participants to indicate, on a 7 -point Likert scale $(1=$ very weak, $7=$ very strong $)$, the level of intensity of 7 emotions related to COVID-19: fear, anger, disgust, hope, anxiety, shock, and feeling of security. We also asked participants to indicate the level of intensity of these 7 emotions evoked by the societal response, defined as the response of Quebecois people, public health organizations, and the government to the COVID-19 situation. Additionally, we asked participants about three more emotions related to the societal response: solidarity, pride, and satisfaction. We added three emotions to the list in phase 2 and phase 3 of the study: frustration about the pandemic, frustration related to the societal response, and solitude. 


\section{Cognitive Reflection Test}

The Cognitive Reflection Test (CRT; Primi et al., 2016) is a test designed to evaluate participants' tendency to override an intuitive but incorrect response in favor of a less intuitive but normally correct response. It consists of a series of 6 mathematical questions such as "A bat and a ball cost $\$ 1.10$ in total. The bat costs $\$ 1.00$ more than the ball. How much does the ball cost?" The score on the CRT is obtained by summing the number of normatively correct responses. A lower score reflects a tendency to reason more intuitively, while a higher score reflects a tendency to reason more systematically. CRT performance is stable across time (Stagnaro \& al., 2018) and is thought to measure reasoning style at the trait level.

\section{Deductive reasoning task}

In phase 1 , responders were assigned to either the deductive reasoning task $(n=292)$ or the base rate task. Both tasks were used to provide an indication of Type 1 and Type 2 processing. In the deductive reasoning task, they were asked to determine whether the conclusion of 16 syllogisms (i.e. All A are B. Some B are C. Therefore, some A are C.) were logically valid or invalid. They were instructed to consider both premises as true and determine whether the conclusion was a necessary outcome of both premises (valid conclusion) or not (invalid conclusion). We manipulated three parameters: Relation to COVID-19 (related, unrelated), logical validity (valid, invalid), and believability of the conclusion (believable, unbelievable).

For the purposes of the current report, we calculated two indexes from this test for each responder. The first was a logic index obtained by determining the percentage of responses that corresponded to the rules of normative logic. A higher value for the logic index indicates more systematic than heuristic reasoning. The second index represented belief bias, defined here as the 
tendency to rely on the believability of a conclusion (instead of its logical structure) to decide whether a syllogism is valid or not (Evans et al., 1983). We obtained this index with the following formula: \% correct responses (congruent trials) - \% correct responses (incongruent trials), where logic and believability favor the same response on congruent trials and different responses on incongruent trials. A higher value for the belief bias index indicates that a responder generally relies on the believability of conclusions, rather than systematic analysis of the structure of the syllogism, in order to evaluate the validity of a syllogism. Therefore, it is an index of Type 1 reasoning.

\section{Base rate tasks}

In phase 1, 238 responders answered 16 base rate problems (Kahneman \& Tversky, 1973). A base rate task with minor differences involving 18 base rate problems was also presented to all participants $(\mathrm{n}=171)$ in the third questionnaire.

Base rate problems follow this structure: "You pick someone randomly in a group of 995 accountants and 5 clowns. You pick Bob. Bob is funny. Bob is more likely to be... a) an accountant, b) a clown." These problems are designed to evaluate base rate neglect. Base rate neglect is defined as a tendency to focus on individual-specific information, such as stereotypes (i.e. Bob is funny), over statistical information (i.e. 995 accountants and 5 clowns). Relying on stereotypes is considered a Type 1 process while combining individual-specific and statistical information is considered a Type 2 process. Base rate tasks are typically designed to unambiguously make the statistical information more reliable than the individual-specific information when the two types of information are incongruent. Thus, a response that is corresponds only with individual-specific information has likely been reached by Type 1 
processes, while a response that corresponds only to statistical information has likely been reached by Type 2 processes.

We manipulated two variables: relation to COVID-19 and the congruency between stereotyped information and base rate information. In phase 1 and phase 3 of the study, we obtained an index of base rate neglect by subtracting the percentage of responses concordant with base rates in incongruent trials from the same percentage in congruent trials. A higher value for the base rate neglect index corresponds to more frequent reliance on stereotypes (Type 1 processes) rather than statistical information (Type 2 processes).

PHQ-9

The depression module of the Patient Health Questionnaire (PHQ-9; Kroenke et al., 2001) is a screening questionnaire consisting of 9 questions about depression symptoms. Responders rated the frequency with which they experienced the 9 symptoms in the last two weeks on a four-point scale from " 0 - never" to " 3 - almost every day." Total scores of 5, 10 and 15 are usually indicative of mild, moderate, and moderately severe depression respectively. We used the French-Canadian version of the test, which shows psychometric properties similar to those of the English version (Arthurs et al., 2012).

$G A D-7$

The Generalized Anxiety Disorder scale (GAD-7; Spitzer et al., 2006) is a screening questionnaire consisting of 7 questions about anxiety symptoms. Responders rated the frequency at which they experienced the 9 symptoms in the last two weeks on a four-point scale from " 0 never" to " 3 - almost every day." Scores of 5, 10 and 15 are usually indicative of mild, moderate, 
and severe generalized anxiety. We used a version of the test adapted for use in a FrenchCanadian population.

\section{Statistical methods}

Statistical methods rely on independent samples t-tests and analyses of correlation. All degrees of freedom reported in t-tests are unadjusted; $\mathrm{t}$ and $\mathrm{p}$ values are adjusted for unequal variances when Levene's test crosses the $p<.05$ threshold. Intra-phase analyses were calculated with data from all participants who responded to the questionnaire. Inter-phase analyses were calculated with data from the 154 participants who responded to all questionnaires. Bonferroni-corrected pvalues are reported for planned contrasts between results from the three phases of the pandemic. All stepwise regressions were calculated with an inclusion criterion of $p<.05$ and an exclusion criterion of $p>.10$. Further specifics for each analysis are reported separately for each research question in the results section.

\section{Results}

\section{Progression of behavior during the pandemic}

Means for presence/absence scores (phase 1) and frequency scores (phases 2 and 3) for different behaviors are available in Table 2. We used paired samples t-tests on multiphase responders' mean behavior frequency scores of phase 2 and phase 3 in order to investigate the impact of the pandemic's progression on pandemic-related behavior. Pandemic-related behavior was less frequent during phase 3 than during phase $2, t(135)=3.16, p=.002, d=0.27$.

Participants complied with public health measures more at the peak of the first wave of the pandemic than during its aftermath, $t(147)=4.59, p<.001, d=0.38$. Looking at the three public health measures of interest separately, we found lowered frequencies for hand washing, $t(153)=$ 
2.33, $p=.021, d=0.19$, and social distancing, $t(147)=6.07, p<.001, d=0.50$, in phase 3, compared to phase 2. However, we found no difference in the efforts not to touch one's face, $t(153)=0.81, p=.425, d=0.060$. There was also an important increase in mask-wearing between phases 2 and 3 of our study, $t(151)=16.66, p<.001, d=1.35$.

$\begin{array}{llll}\text { Behavior } & \text { Phase 1 } & \text { Phase 2 } & \text { Phase 3 } \\ \text { Pandemic-related behavior (mean) } & 0.427(0.142) & 2.44(0.37) & 2.31(0.42) \\ \text { Compliance with public health measures (mean) } & 0.896(0.207) & 3.60(0.46) & 3.42(0.59) \\ \quad \text { More hand washing } & 0.954 & 3.77(0.51) & 3.66(0.69) \\ \quad \text { Social distancing } & 0.974 & 3.90(0.36) & 3.57(0.70) \\ \quad \text { Avoid touching one's face } & 0.760 & 3.14(0.95) & 3.08(0.95) \\ \text { Wearing gloves or a mask } & 0.032 & 1.46(0.80) & 3.00(1.02)\end{array}$

Table 2. Rate of "yes" responses (phase 1) and frequency scores (phases 2 and 3 ) for behavior. Data from responders who returned all questionnaires $(\mathrm{n}=154)$ are provided. Standard deviations are provided in parentheses for all non-dichotomic indexes.

\section{Progression of emotions during the Covid-19 pandemic}

Figure 1 details the progression of emotions during the pandemic. To summarize results, most positive and negative emotions evoked by the pandemic and the societal response became less intense as the pandemic progressed, with two exceptions: feeling of security evoked by the pandemic and disgust evoked by the societal response increased as the pandemic progressed. The most marked decreases ( $F>10$ for phase 1 vs phase 3$)$ were found in fear, anxiety and shock evoked by the pandemic and hope, shock, solidarity, pride and satisfaction evoked by the societal response. 


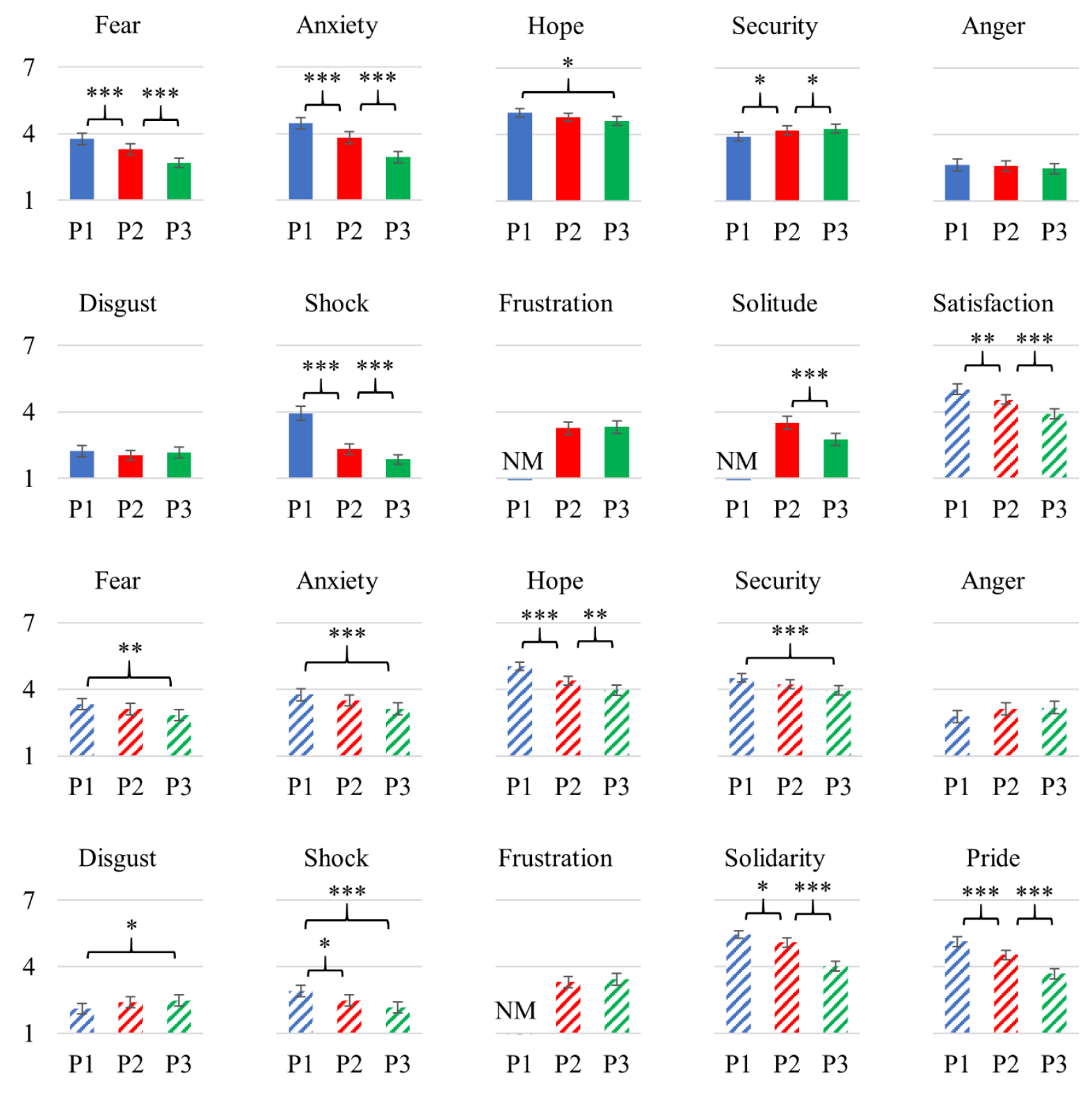

Figure 1. Progression of emotions related to the pandemic (solid bars) and the societal response (striped bars) at three phases of the COVID-19 pandemic. Mean scores on a scale of " 1 - very weak" to "7 - very strong" and unadjusted $95 \%$ confidence intervals for responders who returned all questionnaires $(\mathrm{n}=154)$ are provided. $* p<.05, * * p<.01, * * * p<.001, \mathrm{NM}=$ not measured. Contrasts between phase 1 and phase 3 are not identified as significant if both other contrasts are significant.

\section{Progression of mental health questionnaire scores}


We examined the progression of mental health scores using only participants who responded to all questionnaires $(n=154)$. A paired t-test on PHQ-9 mean scores showed a slight decrease in depression between phase $2(\mathrm{M}=8.38, \mathrm{SD}=4.88)$ and phase $3(\mathrm{M}=7.47, \mathrm{SD}=5.42), t(153)=$ 2.17, $p=.032, d=0.17$. A paired t-test on GAD-7 scores showed a slight decrease in generalized anxiety scores between phase $2(\mathrm{M}=6.95, \mathrm{SD}=4.92)$ and phase $3(\mathrm{M}=6.20, \mathrm{SD}=5.57), t(153)$ $=5.25, p=.023, d=0.19$. Figure 2 shows the distribution of PHQ-9 and GAD-7 scores in phases 2 and 3.

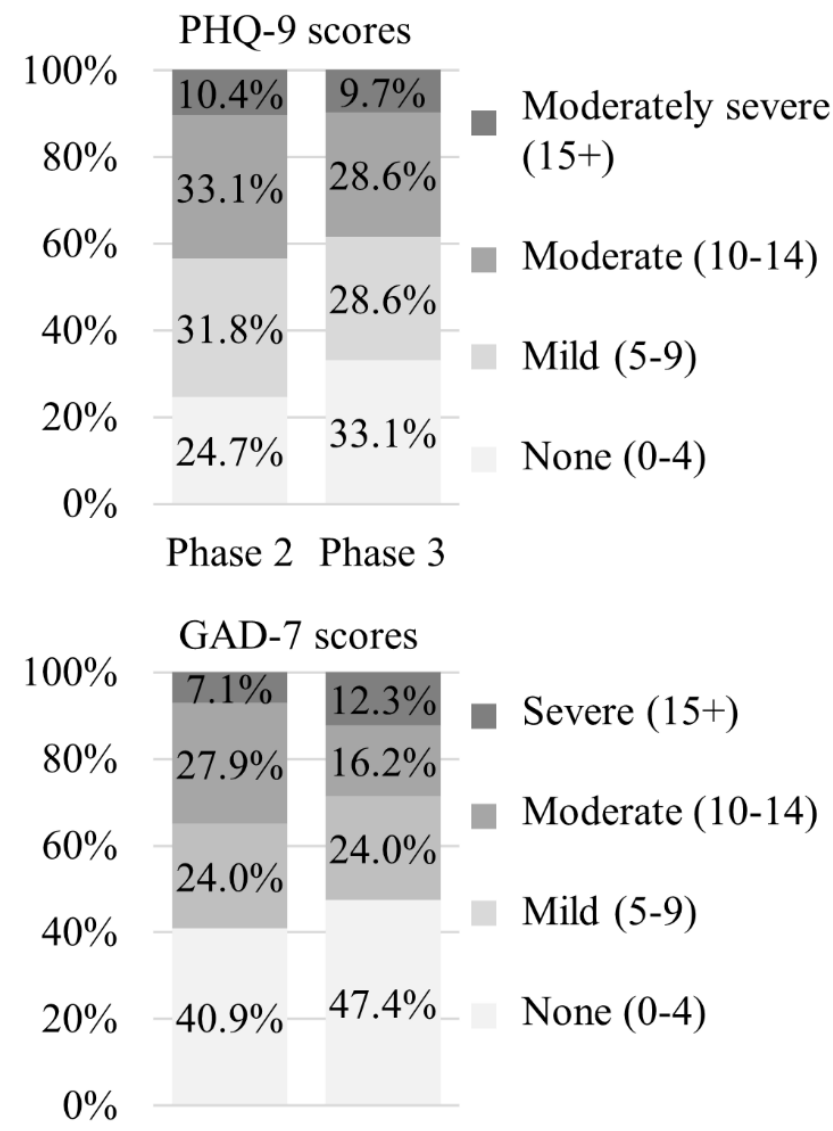

Phase 2 Phase 3

Figure 2. Distribution of PHQ-9 and GAD-7 scores in phases 2 and 3 categorized by their level of severity $(n=154)$.

$\underline{\text { Emotions as predictors of pandemic-related behavior }}$ 
To study emotions as potential predictors of pandemic-related behavior, we processed data from the three phases separately. We entered all emotions into a stepwise regression as independent variables, and the pandemic-related behavior score as the dependent variable. In phase 1, emotions predicted pandemic-related behavior, $F(2,510)=40.80, p<.001, R^{2}=.138$. Stronger fear of the pandemic, $B=0.307, t=6.12, p<.001$, and stronger anxiety evoked by the societal response, $B=0.134, t=2.93, p=.004$, predicted more pandemic-related behavior. In phase 2, emotions predicted pandemic-related behavior, $F(1,199)=19.86, p<.001, R^{2}=.091$. Stronger fear of the pandemic, $B=0.929, t=4.46, p<.001$, was the only significant predictor. In phase 3 , emotions once again predicted pandemic-related behavior, $F(1,150)=52.09, p<.001, R^{2}=.258$. As in phase 2, the only emotional predictor was stronger fear of the pandemic, $B=1.976, t=$ $7.22, p=.001$. To summarize, fear of the pandemic was the most important emotional predictor of pandemic-related behavior in all three phases of the pandemic.

Afterwards, we created an index of change in fear evoked by COVID-19 between phases 2 and 3 by subtracting the score of fear evoked by COVID-19 of phase 2 from the same score in phase 3 . We also created an index of change in the frequency of pandemic-related behaviors by a similar operation. The change indexes of fear and pandemic-related behavior were positively correlated, $r(134)=.206, p=.017$. Participants whose fear rating dropped the most between phases 2 and 3 also tended to be those whose pandemic-related behaviors' frequency was most reduced. Using the same method with scores of other emotions, we found significant correlations between the change indexes of pandemic-related behavior and anxiety evoked by the pandemic, $r(135)=.254$, $p<.003$, and solitude during the pandemic, $r(135)=.275, p=.001$.

\section{$\underline{\text { Emotions as predictors of compliance with public health measures }}$}


To study emotions as potential predictors of compliance with public health measures, we processed data from the three phases separately with stepwise regressions. We entered all emotions as independent variables. Compliance with public health measures was the dependent variable. In phase 1, emotions predicted compliance with public health measures, $F(2,510)=$ $16.14, p<.001, R^{2}=.060$. Stronger fear of the pandemic, $B=0.085, t=4.96, p<.001$, and stronger pride evoked by the societal response, $B=0.046, t=2.57, p=.010$, predicted compliance with public health measures. In phase 2, emotions also predicted compliance with public health measures, $F(2,213)=9.24, p<.001, R^{2}=.080$. Stronger solidarity with the societal response, $B=0.262, t=3.64, p<.001$, and weaker feelings of security evoked by the pandemic, $B=-0.222, t=-3.02, p=.003$, were the two predictors conserved in final the model. In phase 3, emotions once again predicted compliance with public health measures, $F(2,160)=32.14, p<$ $.001, R^{2}=.195$. The two emotional predictors in the final phase 3 model were higher fear of the pandemic, $B=0.578, t=5.67, p=.001$, and higher pride evoked by the societal response, $B=$ $0.204, t=2.36, p=.019$. To summarize these results, avoidance-related emotions evoked by the pandemic and approach-related emotions evoked by the societal response both held predictive power over compliance with public health measures in all three phases of the study.

To investigate links between the progression of emotions and the progression of compliance with public health measures, we created indexes of change in compliance with public health measures by subtracting the phase 2 score from the phase 3 score. No correlation between the change in compliance with public health measures and change scores for emotions was obtained.

\section{$\underline{\text { Reasoning styles as a predictor of behavior }}$}

Correlation coefficients between measures of reasoning and behavior are available in Table 2. To summarize, only one out of five correlations between pandemic-related behavior and reasoning 
measures was statistically significant. It showed a link between low CRT scores indicative of Type 1 reasoning and more pandemic-related behavior. Only one out of five correlations between compliance with public health measures and reasoning measures was statistically significant. It showed a link between high belief bias scores indicative of Type 1 reasoning and more compliance with public measures.

\begin{tabular}{|c|c|c|c|c|}
\hline Measure of reasoning & Phase & $\mathrm{N}$ & $\begin{array}{l}\text { Pandemic- } \\
\text { related behavior }\end{array}$ & $\begin{array}{l}\text { Public health } \\
\text { measures }\end{array}$ \\
\hline CRT & 1 & 526 & $-.096 *(.028)$ & $-.014(.746)$ \\
\hline Logic index (syllogisms) & 1 & 279 & $-.047(.434)$ & $-.106(.078)$ \\
\hline Belief bias index (syllogisms) & 1 & 279 & $.095(.115)$ & $.141 *(.018)$ \\
\hline Base rate neglect index & 1 & 236 & $.061(.349)$ & $-.031(.635)$ \\
\hline Base rate neglect index & 3 & 152 & $.151(.062)$ & $.081(.304)$ \\
\hline
\end{tabular}

Table 2. Correlation coefficients between reasoning measures and behavior measures. All correlations were calculated with reasoning and behavior measures from the same phase of the study. $\mathrm{R}$ values and $\mathrm{p}$ values (in parentheses) are provided.

To investigate whether reasoning predicted behavior if emotions were also considered, we recalculated the regression models detailed above by including a measure of reasoning as a possible predictor of pandemic-related behavior and compliance with public health measures. Performance on the CRT is the only measure of reasoning obtained for all participants, and it is thought to be relatively stable across time; it was therefore included in all six models. Out of six stepwise regressions, only one showed different results than those reported above: The regression model for compliance with public health measures in phase $2, F(3,212)=8.03, p<.001, R^{2}=$ .102 , included lower CRT scores as a third predictor of high compliance, $B=-0.123, t=-.2 .29, p$ $=.023$, after stronger solidarity with the societal response, $B=0.260, t=3.65, p<.001$, and weaker feelings of security related to the pandemic, $B=-0.210, t=-2.87, p=.004$. Reasoning style remained a weaker predictor than avoidance-related and approach-related emotions in this model. 
$\underline{\text { Mental health as a predictor of behavior }}$

Correlations between scores on depression (PHQ-9) and general anxiety (GAD-7) questionnaires and measures of behaviour were calculated in phases 2 and 3 of our study. In phase 2, pandemicrelated behavior correlated with the general anxiety score, $r(206)=.210, p=.002$, but not with the depression score, $r(206)=.050, p=.472$. In phase 3 , the pandemic-related behavior score correlated with scores for anxiety, $r(155)=.272, p=.001$, and depression, $r(155)=.267, p=$ .001 .

However, we observed a trend towards an inverse correlation between depression scores and compliance with public health measures, $r(221)=-.118, p=.80$, in phase 2 of the study. The other three correlations between mental health and compliance with public health measures were non-significant $(p>.10)$.

To verify whether mental health predicted behavior even if emotions were considered, we recalculated the stepwise regression models detailed in the previous section by including generalized anxiety and depression scores in the phase 2 and phase 3 models. Only the final predictive model of compliance with public health measures in phase $2, F(4,211)=6.71, p<$ $.001, R^{2}=.113$, included fewer depression symptoms, $B=-0.038, t=-2.14, p=.027$, as a predictor of higher compliance with public health measures. Thus, there was some evidence that depression symptoms were associated with lower compliance with public health measures even when we accounted for the intensity of several emotions.

\section{Discussion}

We undertook an investigation of behavior, emotions, mental health and reasoning, one week after the beginning of lockdown in the province of Quebec. Unlike other studies undertaken 
simultaneously, we adopted a longitudinal design and asked the same responders to report their emotions and their behavior again at the peak and the aftermath of the first wave of the COVID19 pandemic. Furthermore, we added measures of generalized anxiety and depression during both subsequent phases of the study. Thus, our results allowed us to document the progression of emotions, behavior and mental health throughout the first wave of the COVID-19 pandemic. Our results also allowed us to establish correlations between behavior and measures of emotions, reasoning, and mental health.

We must mention a few limits of the current study before detailing its conclusions. Notably, our convenience sample was younger, more educated, and contained a higher proportion of women than the general population of Quebec from which we drew our sample. Other research undertaken simultaneously to our project has suggested that age (Clark et al., 2020; Haischer et al., 2020; Solomou \& Constantinidou, 2020) and gender (Galasso et al., 2020; Haischer et al., 2020; Solomou \& Constantinidou, 2020) both affect compliance with public health measures. Moreover, it is likely that potential responders self-selected based on their views towards the pandemic, and that those who took the pandemic seriously chose to respond at higher rates than those who did not. Considering these limit, the rates of compliance with public health measures in our sample is likely be unrepresentative of the population of Quebec as a whole. We note that obtaining a nationally representative sample was not our objective and that the progression of emotion and behavior was of principal interest in the current study, not the rates of compliance with public health measures. Our sample remains entirely appropriate to document this progression. Likewise, our sample allows us to establish statistical links between behavior and measures of emotion, reasoning and mental health.

Emotions peaked near the onset of the first wave of the pandemic, not at the peak of excess deaths 
Negative emotions such as fear, anxiety and shock evoked by the pandemic, but also positive emotions such as solidarity, pride, and satisfaction evoked by the societal response, were most intense at the beginning of the pandemic and not at the peak of the first wave. Fear of the pandemic was clearly not linked to the number of weekly excess deaths, an indicator of the current gravity of the situation. These results were generally consistent with an interpretation based on emotional habituation (Frijda, 2007), specifically with the idea that responders' emotions habituated to the hardships of the pandemic even as the pandemic progressed towards its first peak. However, other explanations could be proposed. For instance, it is possible that these crisis-related emotions were mostly anticipatory and that they subsided as uncertainty about the future of the pandemic was reduced.

\section{Avoidance-related emotions as predictors of pandemic-related behavior}

Emotions promoting a motivational tendency towards avoidance of the pandemic predicted pandemic-related behavior and compliance with public health measures at the beginning, at the peak, and in the aftermath of the first wave of the pandemic. Notably, fear of the pandemic was the most important predictor of pandemic-related behavior in all three phases of our study. Responders who reported more fear evoked by COVID-19 tended to report more pandemicrelated behavior. Moreover, fear was the most important emotional predictor of compliance with public health measures in two out of three phases of the study.

Furthermore, the change in fear between the peak of the first wave of the pandemic and its aftermath predicted the degree to which pandemic-related behavior changed between phases. In other words, as fear diminished, the frequency of pandemic-related behavior decreased, including behaviors that were promoted by public health campaigns and those that were not. 
Of course, our data linking avoidance-related emotions and behavior remain correlational and do not demonstrate a causal relationship between emotions and behavior. However, they are entirely consistent with existing theoretical models (Baumeister et al., 2007; Elliot et al., 2013) proposing such a causal link. Moreover, the stability of the observed correlation between fear and behavior across three phases of the pandemic and the co-occurrence of changes in fear and behavior strongly suggest that fear of the pandemic will likely remain an important predictor of behavior in future waves of the pandemic and in other global health crises.

\section{Approach-related emotions predicted compliance with public health measures}

Emotions promoting a motivational tendency towards approach, such as pride and solidarity evoked by the societal response, helped predict compliance with public health measures in all three phases of our study. Higher levels of these emotions were associated with higher compliance with public measures. Importantly, these emotions were only linked to compliance with public health measures, not to all pandemic-related behavior. These results are consistent with the idea that positive feelings evoked by the societal response modulate reactions to public health campaigns (Bogg \& Milad, 2020).

\section{People remained sensitive to new public health guidelines late into the first wave of the pandemic}

Between the peak of the first wave of the pandemic and its aftermath, we observed a small reduction in compliance with public health measures such as hand washing and social distancing. However, these changes were relatively small compared to an important increase in maskwearing behavior during the same period, consistent with changes in public health recommendations. Only $14 \%$ of our responders reported wearing gloves or a mask "sometimes" or "often" at the peak of the first wave of the pandemic, while $72 \%$ of our responders did so 
during the aftermath of the first wave. This increase is likely a result of increased efforts to promote mask wearing by public health authorities. We note that all data was collected before mask-wearing became mandatory in closed public places in the province of Quebec, and that compulsion by authorities is not a possible explanation for this change in behavior. We conclude that despite a clear impact of individual differences in negative emotions on the adoption of pandemic-related behavior and a lowered intensity level for these emotions during the aftermath of the first wave, a high proportion of responders still began complying with a new public health measure late into the crisis.

\section{Reasoning styles had a limited impact on behavior}

One of the objectives of the current study was to investigate links between behavior during the COVID-19 pandemic and two different types of reasoning. Type 1 reasoning tends to be intuitive, automatic and fast, and requires few cognitive resources. Type 2 reasoning tends to be systematic, effortful and slow, and requires many cognitive resources. We hypothesized that Type 2 reasoning would promote adaptive behavior such as compliance with public health measures. However, with five different measures of reasoning distributed across two phases of our study, we found no evidence supporting the hypothesis that Type 2 reasoning led to the adoption of recommended pandemic-related behavior. Some results unexpectedly showed a correlation in the opposite direction, while most results showed that reasoning styles did not predict behavior. We consider the scope of our results too limited to strongly support the conclusion that more Type 1 reasoning was linked to more pandemic-related behavior or more compliance with public health measures. In contrast, our data was not consistent with the hypothesis that Type 2 reasoning promotes compliance with public health measures. However, the fact that our sample was more educated than the general population might impose limits on 
the generalizability of this result. Finally, we also conclude that individual differences in reasoning styles are a much weaker predictor of pandemic-related behavior than individual differences in the emotions elicited by the pandemic.

\section{Poorer mental health scores did not predict lower compliance with public health measures}

Potential links between mental health and behavior were also under study. Two previous reports showed a link between more mental health (generalized anxiety and depression) symptoms and stronger compliance with public health measures (Marroquín, 2020; Stickley et al., 2020). A causal interpretation of these previous reports might suggest that interventions aiming to improve mental health could involuntarily decrease compliance with public health measures. However, our results do not replicate these findings. Instead, we obtained null and contrary results. Responders who reported higher compliance with public health measures also reported fewer depression symptoms, but only at the peak of the first wave of the pandemic. Interestingly, lower levels of depression symptoms remained a predictor of higher compliance with public health measures even when emotions were also included in our regression model. However, compliance with public health measures was not linked to generalized anxiety symptoms. Our results suggest that mental health interventions should not be expected to reduce compliance with public health measures.

Our analyses did suggest that pandemic-related behavior was more frequent for responders who had more generalized anxiety and depression symptoms. However, we detected no effects of mental health on pandemic-related behavior when emotions were also considered. Direct links between mental health and several pandemic-related behaviors could thus be attributable to emotions. 


\section{Conclusion}

Our study contributes to a growing literature on the psychology of human behavior during crises generally, and during the COVID-19 pandemic specifically. Among all studies undertaken simultaneously at the beginning of the COVID-19 pandemic, ours distinguishes itself by its aim to document the progression of emotions, mental health, and behavior throughout different phases of the pandemic. The descriptive accounts of this progression may help predict the time course of emotional responses to future crises.

Our research also underlines the importance of both positive and negative emotions as predictors of inter-individual differences in pandemic-related behavior, and the relatively low predictive value of reasoning style. Whereas emotions such as fear incited people to change their behavior in response to the pandemic, pride and solidarity evoked by the societal response specifically promoted compliance with public health measures. Future directions for research on psychological determinants of behavior in times of crisis may attempt to directly test models proposing that emotions play a causal role in influencing behavior in times of crisis and, if so, investigate whether they act as a mediator between other variables and behavior.

\section{Acknowledgements}

The current project was supported by NSERC grant RGPIN/2019-06384, attributed to Isabelle Blanchette. We have no conflict of interest to report.

\section{References}

Arthurs, E., Steele, R. J., Hudson, M., Baron, M., \& Thombs, B. D. (2012). Are scores on English and French versions of the PHQ-9 comparable? An assessment of differential item functioning. PloS one, 7(12), e52028. https://doi.org/10.1371/journal.pone.0052028 
Baumeister, R. F., Vohs, K. D., Nathan DeWall, C., \& Zhang, L. (2007). How emotion shapes behavior: Feedback, anticipation, and reflection, rather than direct causation. Personality and social psychology review, 11(2), 167-203. https://doi.org/10.1177/1088868307301033

Bogg, T., \& Milad, E. (2020, April 3). Slowing the Spread of COVID-19: Demographic, Personality, and Social Cognition Correlates of Coronavirus Guideline Adherence in a Representative U.S. Sample. Health Psychology, 39(12), 1026-1036. http://dx.doi.org/10.1037/hea0000891

Calvillo, D. P., \& Burgeno, J. N. (2015). Cognitive reflection predicts the acceptance of unfair ultimatum game offers. Judgment \& Decision Making, 10(4).

Capraro, V., \& Barcelo, H. (2020). Priming reasoning increases intentions to wear a face covering to slow down COVID-19 transmission. ArXiv [Working Paper]. https://arxiv.org/abs/2006.11273

Čavojová, V., Šrol, J., \& Mikušková, E. B. (2020). Scientific reasoning as a predictor of healthrelated beliefs and behaviors in the time of COVID-19. PsyArXiv [Working Paper]. https://doi.org/10.31234/osf.io/tfy5q

Clark, C., Davila, A., Regis, M., \& Kraus, S. (2020). Predictors of COVID-19 voluntary compliance behaviors: An international investigation. Global transitions, 2, 76-82. https://doi.org/10.1016/j.glt.2020.06.003

Elliot, A. J., Eder, A. B., \& Harmon-Jones, E. (2013). Approach-avoidance motivation and emotion: Convergence and divergence. Emotion Review, 5(3), 308-311. https://doi.org/10.1177/1754073913477517 
Erceg, N., Ružojčić, M., \& Galic, Z. (2020). Misbehaving in the Corona crisis: the role of anxiety and unfounded beliefs. Current Psychology. https://doi.org/10.1007/s12144-020$\underline{01040-4}$

Evans, J. S. B. (2010). Intuition and reasoning: A dual-process perspective. Psychological Inquiry, 21(4), 313-326. https://doi.org/10.1080/1047840X.2010.521057

Evans, J. S. B., Barston, J. L., \& Pollard, P. (1983). On the conflict between logic and belief in syllogistic reasoning. Memory \& cognition, 11(3), 295-306. https://doi.org/10.3758/BF03196976

Evans, J. S. B., \& Stanovich, K. E. (2013). Dual-process theories of higher cognition: Advancing the debate. Perspectives on psychological science, 8(3), 223-241. https://doi.org/10.1177/1745691612460685

Fitzpatrick, K. M., Harris, C., \& Drawve, G. (2020). Fear of COVID-19 and the mental health consequences in America. Psychological Trauma: Theory, Research, Practice, and Policy. http://dx.doi.org/10.1037/tra0000924

Frijda, N. H. (1988). The laws of emotion. American psychologist, 43(5), 349. https://doi.org/10.1037/0003-066X.43.5.349

Galasso, V., Pons, V., Profeta, P., Becher, M., Brouard, S., \& Foucault, M. (2020). Gender differences in COVID-19 related attitudes and behavior: Evidence from a panel survey in eight OECD countries (No. w27359). National Bureau of Economic Research [Working Paper]. https://doi.org/10.3386/w27359 
Harper, C. A., Satchell, L. P., Fido, D., \& Latzman, R. D. (2020). Functional fear predicts public health compliance in the COVID-19 pandemic. International journal of mental health and addiction. https://doi.org/10.1007/s11469-020-00281-5

Institut de la statistique du Québec. (2020, September 27). Weekly number of deaths in Québec, 2010-2020. https://www.stat.gouv.qc.ca/statistiques/population-demographie/decesmortalite/nombre-hebdomadaire-deces_an.html

Kahneman, D., \& Tversky, A. (1973). On the psychology of prediction. Psychological Review, 80(4), 237. https://doi.org/10.1037/h0034747

Kroenke, K., Spitzer, R. L., \& Williams, J. B. (2001). The PHQ-9: validity of a brief depression severity measure. Journal of general internal medicine, 16(9), 606-613. https://doi.org/10.1046/j.1525-1497.2001.016009606.x

Levine, L. J., \& Bluck, S. (1997). Experienced and remembered emotional intensity in older adults. Psychology and Aging, 12(3), 514-523. https://doi.org/10.1037/0882-7974.12.3.514

Pennycook, G., \& Rand, D. G. (2019). Cognitive reflection and the 2016 US Presidential election. Personality and Social Psychology Bulletin, 45(2), 224-239. https://doi.org/10.1177/0146167218783192

Primi, C., Morsanyi, K., Chiesi, F., Donati, M. A., \& Hamilton, J. (2016). The development and testing of a new version of the cognitive reflection test applying item response theory (IRT). Journal of Behavioral Decision Making, 29(5), 453-469. https://doi.org/10.1002/bdm.1883

Rossi, R., Socci, V., Talevi, D., Mensi, S., Niolu, C., Pacitti, F., Di Marco, A., Rossi, A., Siracusano, A., \& Di Lorenzo, G. (2020). COVID-19 pandemic and lockdown measures 
impact on mental health among the general population in Italy. Frontiers in Psychiatry, 11. https://doi.org/10.3389/fpsyt.2020.00790

Sonnemans, J., \& Frijda, N. H. (1994). The structure of subjective emotional intensity. Cognition \& Emotion, 8(4), 329-350. https://doi.org/10.1080/02699939408408945

Sonnemans, J., \& Frijda, N. H. (1995). The determinants of subjective emotional intensity. Cognition \& Emotion, 9(5), 483-506.

https://doi.org/10.1080/02699939508408977

Solomou, I., \& Constantinidou, F. (2020). Prevalence and predictors of anxiety and depression symptoms during the COVID-19 pandemic and compliance with precautionary measures: Age and sex matter. International journal of environmental research and public health, 17(14), 4924. https://doi.org/10.3390/ijerph17144924

Spitzer, R. L., Kroenke, K., Williams, J. B., \& Löwe, B. (2006). A brief measure for assessing generalized anxiety disorder: the GAD-7. Archives of internal medicine, 166(10), 10921097. https://doi.org/10.1001/archinte.166.10.1092

Stanley, M., Barr, N., Peters, K., \& Seli, P. (2020). Analytic-thinking predicts hoax beliefs and helping behaviors in response to the COVID-19 pandemic. PsyArXiv [Working Paper]. https://doi.org/10.31234/OSF.IO/M3VTH

Stagnaro, M., Pennycook, G., \& Rand, D. G. (2018). Performance on the Cognitive Reflection Test is stable across time. Judgment and Decision Making, 13, 260-267. https://dx.doi.org/10.2139/ssrn.3115809 
Stupple, E., Gale, M., \& Richmond, C. (2013). Working memory, cognitive miserliness and logic as predictors of performance on the cognitive reflection test. In Proceedings of the Annual Meeting of the Cognitive Science society (Vol. 35, No. 35).

Todd, P. M., \& Gigerenzer, G. E. (2012). Ecological rationality: Intelligence in the world. Oxford University Press.

Twenge, J. M., \& Joiner, T. E. (2020). US Census Bureau-assessed prevalence of anxiety and depressive symptoms in 2019 and during the 2020 COVID-19 pandemic. Depression and anxiety. https://doi.org/10.1002/da.23077

Verduyn, P., Van Mechelen, I., Tuerlinckx, F., Meers, K., \& Van Coillie, H. (2009). Intensity profiles of emotional experience over time. Cognition and Emotion, 23(7), 1427-1443. https://doi.org/10.1037/a0014610

Wang, C., Pan, R., Wan, X., Tan, Y., Xu, L., McIntyre, R. S., Choo, F.N., Tran, B., Ho, R., Sharma, V.S. \& Ho, C. (2020). A longitudinal study on the mental health of general population during the COVID-19 epidemic in China. Brain, behavior, and immunity. https://doi.org/10.1016/j.bbi.2020.04.028

Xie, W., Campbell, S., \& Zhang, W. (2020, May 5). Working Memory Capacity Predicts Individual Differences in Social Distancing Compliance during the COVID-19 Pandemic in the U.S. PsyArXiv [Working Paper]. https://doi.org/10.31234/osf.io/3j69f 